PROCEEDINGS OF THE

AMERICAN MATHEMATICAL SOCIETY

Volume 136, Number 12, December 2008, Pages 4257-4266

S 0002-9939(08)09504-X

Article electronically published on July 22, 2008

\title{
GENERIC SUBADDITIVE FUNCTIONS
}

\author{
N. H. BINGHAM AND A. J. OSTASZEWSKI
}

(Communicated by Michael T. Lacey)

\begin{abstract}
We prove a generalization of the 'Subadditive Limit Theorem' and of the corresponding Berz Theorem in a class of functions that includes both the measurable functions and the 'Baire functions'. The generic subadditive functions are defined by a combinatorial property previously introduced by the authors for the study of the foundations of regular variation. By specialization we provide the previously unknown Baire variants of the fundamental theorems of subadditive functions, answering an old question posed by Bingham, Goldie, and Teugels in 1987.
\end{abstract}

\section{INTRODUCTION AND DEFINITIONS}

The class of subadditive functions is interesting from the point of view of both theory and applications. Regarding theory (for which see e.g. Ros, HP, Kucz) they have connections with both additive functions and convex functions. All three classes share pathologies in general (the Hamel pathology; see e.g. [BGT, p. 5]), where they occur in connection with the class of regularly varying functions), but have good properties under minimal regularity assumptions. In BOst1, BOst3, BOst4 we undertook the programme of developing the theory of regular variation under minimal assumptions; the resulting theory of regular variation was there called generic because it gave a common generalization of the measurable and Baire cases (Kech, (8.5), p. 42]). It turns out that the methods developed there lend themselves to the corresponding programme for subadditive functions. Accordingly, we call the resulting theory that of generic subadditive functions. Regarding applications: for analysis, the principal theorem is the (widely used) limit theorem for subadditive functions, generalized below as the 'First Limit Theorem'. This has a probabilistic version, the subadditive ergodic theorem (see King, Lig), an extension of which provided additional motivation for this paper.

We offer here a generalization of the 'Subadditive Limit Theorem' applicable to a class of real-valued subadditive functions defined on $\mathbb{R}^{N}$ that includes both the measurable functions and functions with the Baire property (briefly the Baire functions), namely the class of WNT functions as defined below. This is a less restrictive class of functions permitting a unified treatment of the two classical cases, and still adequate, since, as we show, a sublinear function on $\mathbb{R}^{N}$ is continuous iff it is in WNT. The conclusion that the theorem applies to Baire subadditive

Received by the editors June 29, 2007.

2000 Mathematics Subject Classification. Primary 39B62.

Key words and phrases. No trumps principle, subadditive function.

(C)2008 American Mathematical Society Reverts to public domain 28 years from publication 
functions appears to be new. We extend several fundamental theorems concerning subadditive functions on $\mathbb{R}^{N}$ to the class WNT and prove a Uniform Convergence Theorem as a contribution to the understanding of the Subadditive Limit Theorem. We note that other approaches are known: MatŚw considers one-to-one instead of measurable or Baire.

Perhaps the two main contributions of the paper are:

(i) extension of the Subadditive Limit Theorem to the Baire case (which, as it turns out, is actually the more important of the two cases - see below), and beyond it to WNT;

(ii) the Uniform Convergence Theorem, which seems to be altogether new in this context of subadditivity.

We begin by recalling some combinatorial definitions from BOst3. We follow the set-theorists and denote the set of natural numbers by $\omega=\{0,1,2, \ldots\}$.

Definition 1. For a family $\left\{T_{k}: k \in \omega\right\}$ of subsets of $\mathbb{R}^{N}, \mathbf{N T}\left(\left\{T_{k}: k \in \omega\right\}\right)$ means that, for every bounded/convergent sequence $\left\{\mathbf{u}_{n}\right\}$ in $\mathbb{R}^{N}$, some $T_{k}$ contains a translate of a subsequence of $\left\{\mathbf{u}_{n}\right\}$; i.e. there is $k \in \omega$, infinite $\mathbb{M} \subseteq \omega, \mathbf{t} \in \mathbb{R}^{N}$ such that

$$
\left\{\mathbf{t}+\mathbf{u}_{n}: n \in \mathbb{M}\right\} \subseteq T_{k} .
$$

For the function $h: \mathbb{R}^{N} \rightarrow \mathbb{R}$, its (symmetric) level sets are defined by

$$
H^{r}, \text { or } H^{r}(h),:=\left\{\mathbf{x} \in \mathbb{R}^{N}:|h(\mathbf{x})|<r\right\} .
$$

Definition 2 (WNT-functions). (1) Let $h: \mathbb{R}^{N} \rightarrow \mathbb{R}$. We call $h$ a WNTfunction, $h \in \mathbf{W N T}$, if $\mathbf{N T}\left(\left\{H^{k}: k \in \omega\right\}\right)$ holds.

(2) Let $h: \mathbb{R}^{N} \rightarrow[-\infty, \infty]$. We call $h$ a WNT-function, $h \in \mathbf{W N T}$, if $\mathbf{N T}\left(\left\{H^{k}: k \in \omega\right\} \cup H^{ \pm \infty}\right)$ holds, where $H^{ \pm \infty}=\left\{\mathbf{x} \in \mathbb{R}^{N}:|h(\mathbf{x})|=\infty\right\}$.

Theorem 1 (No Trumps Theorem (Csiszár and Erdös CsEr]; see BOst1])). If T is an interval and $T=\bigcup_{k \in \omega} T_{k}$ with each $T_{k}$ measurable/Baire, then $\boldsymbol{N T}\left(\left\{T_{k}: k \in\right.\right.$ $\omega\})$ holds.

Corollary 1. If $h$ is measurable/Baire, then $h \in \boldsymbol{W N T}$.

Proof. Take $T=\mathbb{R}, T_{k}=H^{k}$ measurable/Baire, and note $\mathbb{R}=\bigcup_{k \in \omega} H^{k}$.

Here, there are two versions of the theorem, for the measurable and Baire cases. Historically, the measurable case came first, and hence has taken precedence. It is now known how to subsume these two cases into one, by means of a 'duality'; see BOst11]. (The density topology allows the deduction of the measurable case from the Baire case, an instance of 'bitopology'.) It is clear from this that it is the Baire case that is in fact the more fundamental, as it includes the measurable case.

\section{Subadditive Limit theorem}

We begin by extending the basic limit theorem for subadditive functions (Rosenbaum Ros, Hille and Phillips [HP, p. 255]).

Theorem 2 (Subadditive Limit Theorem (First Limit Theorem, at Infinity)). If the subadditive function $f: \mathbb{R}^{N} \rightarrow \mathbb{R}$ is in $\boldsymbol{W N T}$, then

$$
F(\mathbf{x}):=\lim _{s \rightarrow+\infty} \frac{f(s \mathbf{x})}{s}, \text { for } \mathbf{x} \in \mathbb{R}^{N},
$$


is finite, positively homogeneous, convex and continuous. Moreover,

$$
F(\mathbf{x})=\inf _{t>0} \frac{f(t \mathbf{x})}{t} .
$$

Remark 1. For $\mathbf{x} \neq 0$, write $v(\mathbf{x})=\mathbf{x} /\|\mathbf{x}\|$; then

$$
F(\mathbf{x})=\|\mathbf{x}\| \lim _{s \rightarrow+\infty} \frac{f(s v(\mathbf{x}))}{s}=\|\mathbf{x}\| F(v(\mathbf{x})), \text { for } \mathbf{x} \in \mathbb{R}^{N} .
$$

Note that, as $F$ is in particular sublinear (subadditive and also $F(n \mathbf{x})=n F(\mathbf{x})$ for $n \in \omega$ ), this formula is in agreement with the theorem of Berz (see below) for measurable sublinear $F$ ([Kucz, p. 415]). We will later see circumstances under which this convergence is uniform. For the meantime we note:

Corollary 2. The Limit Theorem at Infinity holds if the subadditive function $f$ is measurable/Baire.

We draw on some auxiliary results; for proofs see e.g. [Kucz, p. 404].

Lemma 1. For any subadditive function $f: \mathbb{R}^{N} \rightarrow \mathbb{R}$, if $f$ is locally bounded above at a point, then it is locally bounded at every point.

Proposition 1 (Cf. [Kucz, p. 404, Th. 3]). If $f: \mathbb{R}^{N} \rightarrow \mathbb{R}$ in WNT is subadditive, then $f$ is locally bounded.

Proof. By assumption, $\mathbf{N T}\left(\left\{H^{k}: k \in \omega\right\}\right)$ holds for $H^{k}=\{\mathbf{x}:|f(\mathbf{x})|<k\}$. Suppose that $f$ is not locally bounded; then it is not locally bounded above at some point $\mathbf{u}$; i.e. there exists $\mathbf{u}_{n} \rightarrow \mathbf{u}$ with $f\left(\mathbf{u}_{n}\right) \rightarrow+\infty$. For some $k \in \omega$, $\mathbf{t} \in \mathbb{R}^{N}$ and an infinite $\mathbb{M}$ we have $\left\{\mathbf{t}+\mathbf{u}_{n}: n \in \mathbb{M}\right\} \subseteq H^{k}$. For $n$ in $\mathbb{M}$, we have $f\left(\mathbf{u}_{n}\right)=f\left(\mathbf{t}+\mathbf{u}_{n}-\mathbf{t}\right) \leq f\left(\mathbf{t}+\mathbf{u}_{n}\right)+f(-\mathbf{t}) \leq k+f(-\mathbf{t})$, which contradicts $f\left(\mathbf{u}_{n}\right) \rightarrow+\infty$.

Proof of the First Limit Theorem. Fix $\mathbf{x} \neq 0$. Put

$$
\beta=\beta_{+}(\mathbf{x})=\inf _{t>0} f(t \mathbf{x}) / t \geq-\infty .
$$

(We have adapted the notation of [HP] for clarity and to suit later needs.) As $\beta<\infty$, consider $b \in \mathbb{R}$ with $b>\beta$ and select $t_{0}$ with $f\left(t_{0} \mathbf{x}\right) / t_{0}<b$. Now consider any $t>3 t_{0}$ and let $m=\left[t / t_{0}\right]$. Thus $m t_{0} \leq t<(m+1) t_{0}$.

As $f$ is WNT, we may, by Proposition 1 , select an $M$ which bounds $f$ on the interval $\left[2 t_{0} \mathbf{x}, 3 t_{0} \mathbf{x}\right]$. Writing $n=m-2$, we have

$$
2 t_{0} \leq t-(m-2) t_{0}=t-n t_{0} \leq 3 t_{0}
$$

Hence, by subadditivity, since $f(n \mathbf{z}) \leq n f(\mathbf{z})$, we have

$$
f(t \mathbf{x})=f\left(n t_{0} \mathbf{x}\right)+f\left(\left(t-n t_{0}\right) \mathbf{x}\right) \leq n f\left(t_{0} \mathbf{x}\right)+f\left(\left(t-n t_{0}\right) \mathbf{x}\right) \leq n f\left(t_{0} \mathbf{x}\right)+M,
$$

and so

$$
\beta \leq \frac{f(t \mathbf{x})}{t} \leq \frac{n t_{0}}{t} \frac{f\left(t_{0} \mathbf{x}\right)}{t_{0}}+\frac{M}{t} \leq \frac{n t_{0}}{t} b+\frac{M}{t} .
$$

But

$$
(m-2) /(m+1) \leq(m-2) t_{0} / t \leq(m-2) / m ;
$$

hence, as $t \rightarrow \infty$, we see that $n t_{0} / t \rightarrow 1$. So

$$
\beta \leq \liminf _{t \rightarrow \infty} f(t \mathbf{x}) / t \leq \limsup _{t \rightarrow \infty} f(t \mathbf{x}) / t \leq b .
$$


But $b>\beta$ was arbitrary, so $\beta=\lim _{t \rightarrow \infty} f(t \mathbf{x}) / t=F(\mathbf{x})<\infty$. Now let $\alpha=$ $\alpha_{-}(\mathbf{x})=\sup _{t<0} f(t \mathbf{x}) / t \leq \infty$. Evidently $\alpha>-\infty$. Substituting $t=-s$, we have

$$
\alpha=-\inf _{s>0} \frac{f(-s \mathbf{x})}{s}=-F(-\mathbf{x})>-\infty .
$$

But, since $f(t \mathbf{x})+f(-t \mathbf{x}) \geq f(t \mathbf{x}-t \mathbf{x})=f(\mathbf{0}) \geq 0$, we have

$$
F(\mathbf{x})+F(-\mathbf{x})=\lim _{t \rightarrow \infty}\left(\frac{f(t \mathbf{x})}{t}+\frac{f(-t \mathbf{x})}{t}\right) \geq 0 .
$$

Thus $-\infty<-F(-\mathbf{x}) \leq F(\mathbf{x})$. So $F(\mathbf{x})$ is finite. Clearly $F$ is positively homogeneous. Moreover, $F$ is subadditive; indeed $f(t \mathbf{x})+f(t \mathbf{y}) \geq f(t(\mathbf{x}+\mathbf{y}))$, so

$$
F(\mathbf{x}+\mathbf{y})=\lim _{t \rightarrow \infty}\left(\frac{f(t(\mathbf{x}+\mathbf{y}))}{t}\right) \leq \lim _{t \rightarrow \infty}\left(\frac{f(t \mathbf{x})}{t}+\frac{f(t \mathbf{y})}{t}\right)=F(\mathbf{x})+F(\mathbf{y})
$$

Hence $F$ is convex: indeed, since it is subadditive and positively homogeneous, we have

$$
F(r \mathbf{u}+s \mathbf{v}) \leq F(r \mathbf{u})+F(s \mathbf{v})=r F(\mathbf{u})+s F(\mathbf{v}),
$$

for $r, s \geq 0$. So ([Rock, Cor. 10.1.1] or [Kucz, Th. 7.1.1, p. 149]) $F$ is continuous.

Example 1. The renewal function of probability theory is always subadditive (and measurable); see [Dal, Section 4], [Fell, Ch. XI]. If $\mu$ is the mean of the lifetime distribution $F$ (of lightbulbs, say), then the renewal function

$$
U(x):=\sum_{n=0}^{\infty} F^{* n}(x)
$$

(here $*$ is convolution and $U(x)$ is the expected number of bulbs needed by time $x$ ) satisfies

$$
U(x+h)-U(x) \sim h / \mu \quad(x \rightarrow \infty) .
$$

In particular, we have a nice illustration of the Limit Theorem at Infinity:

$$
U(x) / x \sim 1 / \mu \quad(x \rightarrow \infty) .
$$

We will need the following result in the next section.

Proposition 2 (Cf. Kucz, p. 406, Th. 7]). Let $f: \mathbb{R}^{N} \rightarrow[-\infty, \infty]$ in $\boldsymbol{W N T}$ be subadditive. Put $\lambda=\liminf _{\mathbf{x} \rightarrow \mathbf{0}} f(\mathbf{x})$. Then $\lambda \geq 0$, or $\lambda= \pm \infty$. If $|\lambda|=+\infty$, then $f$ is infinitary (takes at least one of the values $\pm \infty$ ).

Proof. For some null-sequence $\mathbf{u}_{n}$ we have $f\left(\mathbf{u}_{n}\right) \rightarrow \lambda$. For some $k \in \omega$, an infinite $\mathbb{M}$ and some $\mathbf{t}$, we have $\left\{\mathbf{t}+\mathbf{u}_{n}: n \in \mathbb{M}\right\} \subseteq H^{k}$. For $n$ in $\mathbb{M}$, we have again $f\left(\mathbf{u}_{n}\right)=f\left(\mathbf{t}+\mathbf{u}_{n}-\mathbf{t}\right) \leq f\left(\mathbf{t}+\mathbf{u}_{n}\right)+f(-\mathbf{t}) \leq k+f(-\mathbf{t})$. Passing to the limit, we have $\lambda \leq k+f(-\mathbf{t})$.

Thus if $\lambda=+\infty$, the function $f$ is infinitary at $-\mathbf{t}$. Also, since $f(2 \mathbf{u}) \leq 2 f(\mathbf{u})$, we have

$$
\lambda \leq \liminf f\left(2 \mathbf{u}_{n}\right) \leq \liminf 2 f\left(\mathbf{u}_{n}\right)=2 \liminf f\left(\mathbf{u}_{n}\right)=2 \lambda,
$$

so for $\lambda$ finite we conclude $\lambda \geq 0$. If $f$ assumes only finite values, then it is locally bounded by Proposition 1 , and so $\lambda \neq \pm \infty$. Hence if $|\lambda|=\infty$, the function $f$ is infinitary. 
If $f$ is infinitary it may assume either or both the values $\pm \infty$. If the subadditive $f$ does not assume the value $\infty$, suppose that $f(\mathbf{t})=-\infty$. Then, for all $\mathbf{x}$, we have

$$
f(\mathbf{x})=f(\mathbf{x}-\mathbf{t}+\mathbf{t}) \leq f(\mathbf{x}-\mathbf{t})+f(\mathbf{t})=-\infty .
$$

(Here for each $k \in \omega$, the level sets $H^{k}(f)$ are empty.) On the other hand, the subadditive function $f$, with $f(t)=1$ if $t \in \mathbb{R} \backslash \mathbb{Q}$ and $+\infty$ otherwise, is in WNT and does not assume the value $-\infty$. See [HP, p. 240] for other examples.

Our last result, clarifying the behaviour of subadditive functions taking negative values, mimicks its classical counterpart. For subadditive $f$, recall that $f(\mathbf{0}) \geq 0$.

Theorem 3 (Cf. [HP p. 243] and [Kucz, p. 406, Th. 8]). Let $f: \mathbb{R}^{N} \rightarrow \mathbb{R}$ be subadditive and in $\boldsymbol{W N T}$. Suppose that there exists $\mathbf{x}_{0} \in \mathbb{R}^{N}$ s.t. $f\left(\mathbf{x}_{0}\right)<0$. Then $f\left(t \mathbf{x}_{0}\right)<0$ for all $t>0$ sufficiently large and $f\left(t \mathbf{x}_{0}\right) \geq 0$ for all $t \leq 0$.

Proof. Adapt the proof in $\mathrm{Kucz}$, replacing his Th. 6 by a compactness argument.

\section{Sublinear functions: Berz's theorem}

For this section recall that a sublinear function is subadditive and satisfies

$$
f(n \mathbf{x})=n f(\mathbf{x}), \text { for } n=1,2, \ldots
$$

Such functions are characterized by Berz's Theorem ([Berz, see below). We note, as a canonical example, that the norm function is sublinear. For continuous sublinear functions, it turns out that $f(\mathbf{0})=0$, so the alternative definition that $f(n \mathbf{x})=$ $n f(\mathbf{x})$, for $n \in \omega$, turns out to be equivalent.

The characterization theorem is usually deduced from Berz's Lemma, as quoted below, by way of an additive minorant lemma for measurable functions (see Kucz, p. 218], for the convex minorant lemma). Such a proof is also possible in our context, since the corresponding additive minorant lemma holds also for WNT functions. However, we prefer to deduce the characterization theorem from Berz's Lemma by way of the First Limit Theorem, as then the relevant majorization is explicitly by way of a linear function.

Lemma 2 (Additive Minorant Lemma). Suppose that $f, g: \mathbb{R}^{N} \rightarrow \mathbb{R}$ satisfy

$$
g(\mathbf{x}) \leq f(\mathbf{x}) \text { for all } \mathbf{x} \in \mathbb{R}^{N},
$$

with $g$ additive and $f$ a $\boldsymbol{W N T}$ function. Then $g$ is linear and continuous.

Proof. Suppose not. Then, by Ostrowski's Lemma (see e.g. [BGT, Lemma 1.1.6, p. 4] and BOst3] $)$, there is a convergent sequence $\mathbf{u}_{n}$ with $g\left(\mathbf{u}_{n}\right)$ unbounded from above. For some $\mathbf{t}$ and infinite $\mathbb{M}$ we have $\left\{\mathbf{t}+\mathbf{u}_{m}: m \in \mathbb{M}\right\} \subseteq H^{k}=\{\mathbf{x}:|f(\mathbf{x})|<$ $k\}$. Hence, for $m \in \mathbb{M}$, we have $g(\mathbf{t})+g\left(\mathbf{u}_{m}\right)=g\left(\mathbf{t}+\mathbf{u}_{m}\right) \leq f\left(\mathbf{t}+\mathbf{u}_{m}\right)<k$, a contradiction.

Corollary 3 (Ostrowski's Theorem). A WNT additive function is continuous.

Proof. Take $f=g$.

Lemma 3 (Subadditive Majorant Lemma). For $T \subseteq \mathbb{R}^{N}$ suppose that $\boldsymbol{N T}(T)$ holds (e.g. if $T$ is non-empty and open) and that $f, g: \mathbb{R}^{N} \rightarrow \mathbb{R}$ satisfy

$$
g(\mathbf{x}) \leq f(\mathbf{x}) \text { for } \mathbf{x} \in T,
$$

with $g$ additive, and $f$ a subadditive $\boldsymbol{W N T}$ function. Then $g$ is linear and continuous. 
Proof. Suppose not. Then there is a convergent sequence $\mathbf{u}_{n}$ with $g\left(\mathbf{u}_{n}\right)$ unbounded from above. For some $\mathbf{t}$ and infinite $\mathbb{M} \subseteq \omega$ we have $\left\{\mathbf{t}+\mathbf{u}_{m}: m \in \mathbb{M}\right\} \subseteq T$. As $\left\{\mathbf{t}+\mathbf{u}_{m}: m \in \mathbb{M}\right\}$ is convergent there are $\mathbf{z} \in \mathbb{R}^{N}$, some $k \in \omega$ and an infinite $\mathbb{M}^{\prime} \subseteq \mathbb{M}$ such that $\left\{\mathbf{z}+\mathbf{t}+\mathbf{u}_{m}: m \in \mathbb{M}^{\prime}\right\} \subseteq H^{k}=\{\mathbf{x}:|f(\mathbf{x})|<k\}$. Hence, for $m \in \mathbb{M}^{\prime}$, we have

$$
g\left(\mathbf{t}+\mathbf{u}_{m}\right) \leq f\left(\mathbf{t}+\mathbf{u}_{m}\right) \text { and }\left|f\left(\mathbf{z}+\mathbf{t}+\mathbf{u}_{m}\right)\right|<k .
$$

Thus, for $m \in \mathbb{M}^{\prime}$, since $\mathbf{t}+\mathbf{u}_{m} \in T$, we have

$$
\begin{aligned}
g\left(\mathbf{u}_{m}\right) & =g\left(\mathbf{t}+\mathbf{u}_{m}\right)-g(\mathbf{t}) \leq f\left(\mathbf{t}+\mathbf{u}_{m}\right)-g(\mathbf{t}) \leq f\left(\mathbf{z}+\mathbf{t}+\mathbf{u}_{m}\right)+f(-\mathbf{z})-g(\mathbf{t}) \\
& \leq k+f(-\mathbf{z})-g(\mathbf{t}),
\end{aligned}
$$

as $\mathbf{z}+\mathbf{t}+\mathbf{u}_{m} \in H^{k}$, and so $g\left(\mathbf{u}_{m}\right)$ is bounded, a contradiction.

We now recall a lemma due to Berz; for a proof see e.g. [Kucz, p. 414].

Lemma 4 (Berz's Lemma Berz). For $f: \mathbb{R}^{N} \rightarrow \mathbb{R}$ sublinear,

$$
f(\mathbf{x})=\sup \left\{g(\mathbf{x}) \mid g: \mathbb{R}^{N} \rightarrow \mathbb{R} \text { additive and } g \leq f\right\} .
$$

Theorem 4 (Berz's Theorem [Berz]). For sublinear $f: \mathbb{R}^{N} \rightarrow \mathbb{R}$ in WNT:

$$
f(\mathbf{x})=\|\mathbf{x}\| f(v(\mathbf{x}))=\|\mathbf{x}\| F(v(\mathbf{x})), \text { for } \mathbf{x} \neq 0,
$$

where $F$ is the limit function defined by (2.1). Thus $f$ is positively homogeneous, so $f(\mathbf{0})=0$, and $f$ is continuous.

Proof. Fix $\mathbf{x} \neq \mathbf{0}$. For any additive $h \leq f$, define $g_{h}(t)=h(t \mathbf{x})$. Then, $g_{h}: \mathbb{R} \rightarrow \mathbb{R}$ is additive. Let $\varepsilon>0$. By the First Limit Theorem we have, for all $t>0$,

$$
F(v(\mathbf{x})) \leq f(t \mathbf{x}) / t \text {, i.e. } F(v(\mathbf{x})) t \leq f(t \mathbf{x}),
$$

and also for some $t_{0}>0$ we have, for $t \geq t_{0}$, that

$$
F(v(\mathbf{x})) \leq f(t \mathbf{x}) / t \leq F(v(\mathbf{x}))+\varepsilon .
$$

Thus, for $t \geq t_{0}$, we have $g_{h}(t)=h(t \mathbf{x}) \leq f(t \mathbf{x}) \leq t(F(v(\mathbf{x}))+\varepsilon)$. Thus the additive function $g_{h}$ is locally bounded above far enough to the right by a linear form, and so, by the Subadditive Majorant Lemma, is continuous. Write $g_{h}(t)=c_{h}(\mathbf{x}) t$. Thus we now have, for all $t$, that $c_{h}(\mathbf{x}) t=g_{h}(t)=h(t \mathbf{x}) \leq f(t \mathbf{x})$. It follows from (3.3) that $c_{h}(\mathbf{x}) \leq F(v(\mathbf{x}))$. Hence, by the Berz Lemma, we have for fixed $t \geq 0$ that

$$
f(t \mathbf{x})=\sup \left\{c_{h}(\mathbf{x}) t \mid c_{h}(\mathbf{x}) s \leq f(s \mathbf{x}) \text { all } s\right\} \leq t F(v(\mathbf{x})) .
$$

From here and (3.2) we have, as asserted, that

$$
f(t \mathbf{x})=t F(v(\mathbf{x})) \text { for } t \geq 0 .
$$

For $\mathbf{x}$ a unit vector, so $\mathbf{x}=v(\mathbf{x})$, we obtain $F(v(\mathbf{x}))=f(v(\mathbf{x}))$, and hence (3.4) implies

$$
f(\mathbf{x})=f(\|x\| v(x))=\|x\| F(v(\mathbf{x})) \text { for } t \geq 0,
$$

whence (3.1) and positive homogeneity. Since $v$ is continuous in $\mathbb{R}^{N} \backslash\{\mathbf{0}\}$ and $F$ is continuous, so is $f$.

Theorem 5 (Equivalence Theorem). For $f$ sublinear, $f$ is continuous iff $f \in$ WNT.

Proof. Use Berz's Theorem: continuous functions are in WNT. 
Remark 2. In the sublinear case, even if the definition does not require so, $f(\mathbf{0})=0$, by Berz's Theorem. By contrast, a general subadditive function $f$ in WNT may satisfy $f(\mathbf{0})=0$, be locally bounded and yet not continuous. Proposition 2 puts this in perspective. But such a function $f$ is continuous iff it is continuous at the origin. (See [Kucz, Th. 1, p. 404], or [HP, p. 247].)

\section{Differentiability, Lipschitz COndition, uniform CONVERGEnCE}

Several fundamental theorems on subadditive functions concerning differentiability (see e.g. K Kucz, Ch. XVI] or [HP, Ch. VII] for a review of these) remain valid when measurability is replaced by membership of the class WNT. We note some examples in this section and sketch the proofs where these differ in a significant detail from the classical setting; we refer to results and ideas of the last section. We begin by extending the notation of the Subadditive Limit Theorem to take in the following quantities with which we are concerned in this section, namely

$$
\beta_{-}(\mathbf{x})=\inf _{t<0} f(t \mathbf{x}) / t, \quad \alpha_{+}(\mathbf{x})=\sup _{t>0} f(t \mathbf{x}) / t,
$$

where

$$
-\infty \leq \beta_{-}(\mathbf{x})<\infty \text { and }-\infty<\alpha_{+}(\mathbf{x}) \leq \infty .
$$

Theorem 6 (Second Limit Theorem, at Zero). Let $f: \mathbb{R}^{N} \rightarrow \mathbb{R}$ in $\boldsymbol{W N T}$ be subadditive. Then

(1) $\beta_{-}(\mathbf{x}) \leq-\beta_{-}(-\mathbf{x})=\sup _{t>0} f(t \mathbf{x}) / t=\alpha_{+}(\mathbf{x})$, where the left-hand side may be $-\infty$ and the right $+\infty$.

(2) If $\beta_{-}(\mathbf{x})$ is finite, then $\beta_{-}(\mathbf{x})=G(\mathbf{x}):=\lim _{t \rightarrow 0-} f(t \mathbf{x}) / t$. Similarly, if $-\beta_{-}(-\mathbf{x})$ is finite, then

$$
-\beta_{-}(-\mathbf{x})=-G(-\mathbf{x})=\lim _{t \rightarrow 0+} f(t \mathbf{x}) / t .
$$

(3) The equation in (2) above is also valid under either of the hypotheses

$$
\lim _{x \rightarrow 0} f(\mathbf{x})=0 \text {, or } \lim _{x \rightarrow 0} \inf _{x \rightarrow} f(\mathbf{x})>0 .
$$

(4) If $G(\mathbf{x})$ is well-defined for all $\mathbf{x}$ (i.e. $\beta_{-}(\mathbf{x})$ is finite for all $\left.\mathbf{x}\right)$, then $G(\mathbf{x})$ is positively homogeneous and subadditive, hence convex and continuous.

Proof. The proof of (i)-(iii) depends on Proposition 2 that $\lambda=\liminf _{\mathbf{x} \rightarrow \mathbf{0}} f(\mathbf{x}) \geq 0$ and may be taken verbatim from [Kucz, p. 410]. Now note, as in the Subadditive Limit Theorem, that $\beta_{-}(\mathbf{x})$ is positively homogeneous, so (iv) follows from (ii) and a calculation of subadditivity similar to that in (2.2).

Definition 3. The four Dini derivatives (upper-right, upper-left, etc.) in direction $\mathbf{h}$ are as follows:

$$
\begin{aligned}
D_{\mathbf{h}}^{+} f(\mathbf{x})=\limsup _{t \rightarrow 0+} \frac{f(\mathbf{x}+t \mathbf{h})-f(\mathbf{x})}{t}, & D_{\mathbf{h}}^{-} f(\mathbf{x})=\limsup _{t \rightarrow 0-} \frac{f(\mathbf{x}+t \mathbf{h})-f(\mathbf{x})}{t}, \\
d_{\mathbf{h}}^{+} f(\mathbf{x})=\liminf _{t \rightarrow 0+} \frac{f(\mathbf{x}+t \mathbf{h})-f(\mathbf{x})}{t}, & d_{\mathbf{h}}^{-} f(\mathbf{x})=\liminf _{t \rightarrow 0-} \frac{f(\mathbf{x}+t \mathbf{h})-f(\mathbf{x})}{t} .
\end{aligned}
$$

The notation here, adapted from Kuczma Kucz, is more convenient for identifying directions than that in $[\mathrm{HP}$.

Theorem 7 (Theorem on Dini derivative bounds, [HP, p. 251]). Let $f: \mathbb{R}^{N} \rightarrow \mathbb{R}$ in $\boldsymbol{W N T}$ be subadditive. Then 
(1) The Dini derivatives are bounded as follows:

$$
\begin{aligned}
D_{\mathbf{h}}^{+} f(\mathbf{x}) & \leq-\beta_{-}(-\mathbf{h}), \quad D_{\mathbf{h}}^{-} f(\mathbf{x}) \leq-\beta_{-}(-\mathbf{h}), \\
\beta_{-}(\mathbf{h}) & \leq d_{\mathbf{h}}^{+} f(\mathbf{x}), \quad \beta_{-}(\mathbf{h}) \leq d_{\mathbf{h}}^{-} f(\mathbf{x}) .
\end{aligned}
$$

(2) If $\beta_{-}(\mathbf{x})=-\beta_{-}(-\mathbf{x})$, then $f(t \mathbf{x})=\beta_{-}(\mathbf{x}) t$.

Proof. (i) Only the finite-valued versions require checking. All four cases require an identical approach, so we do just the first. Evidently, $f(\mathbf{x}+t \mathbf{h}) \leq f(\mathbf{x})+f(t \mathbf{h})$, so for $t>0$ we have

$$
\frac{f(\mathbf{x}+t \mathbf{h})-f(\mathbf{x})}{t} \leq \frac{f(t \mathbf{h})}{t} .
$$

But if $\beta(-\mathbf{x}) \neq-\infty$, then $-\beta(-\mathbf{x})$ is finite, so by the last theorem we have

$$
D_{\mathbf{h}}^{+} f(\mathbf{x})=\limsup _{t \rightarrow 0+} \frac{f(\mathbf{x}+t \mathbf{h})-f(\mathbf{x})}{t} \leq \lim _{t \rightarrow 0+} \frac{f(t \mathbf{h})}{t}=-\beta_{-}(-\mathbf{h}) .
$$

(ii) If $\beta(\mathbf{x})=-\beta(-\mathbf{x})$, then both these quantities are finite. By positive homogeneity, $\beta(t \mathbf{x})=t \beta(\mathbf{x})$ for all $t$. Hence $f$ restricted to the linear span of $\mathbf{x}$ is differentiable and $d f(t \mathbf{x}) /\left.d t\right|_{t=0}=\beta_{-}(\mathbf{x})$. So, for some constant $c$, we have

$$
f(s \mathbf{x})=s \beta_{-}(\mathbf{x})+c .
$$

But, if $c \neq 0$, suppose w.l.o.g. that $c>0$. Then we have the contradiction

$$
\beta_{-}(\mathbf{x})=\lim _{t \rightarrow 0-} \frac{f(t \mathbf{x})}{t}=\lim _{t \rightarrow 0-}\left(\beta_{-}(\mathbf{x})+\frac{c}{t}\right)=-\infty .
$$

Theorem 8 (The Lipschitz condition). Let $f: \mathbb{R}^{N} \rightarrow \mathbb{R}$ in WNT be subadditive. Suppose also that $\beta_{-}(\mathbf{x})$ is finite for all $\mathbf{x}$. Then $f$ is a Lipschitz function with constant $L$ provided

$$
L>\sup \{|G(\mathbf{h})|:\|\mathbf{h}\|=1\},
$$

with $G$ as in Theorem 6.

Proof. In view of the Second Limit Theorem, part (iv), under the current circumstances $G$ is a continuous function. Fix a direction $\mathbf{h}$ and a number $L$ satisfying the condition (4.2). Let $\varepsilon=L-\sup \{|G(\mathbf{h})|:|| \mathbf{h} \|=1\}$. Then $\varepsilon>0$. Let $V=\operatorname{span}\{\mathbf{h}\}$. We now follow [Kucz, p. 413]. As in (4.1), given $\mathbf{x} \in V$, we have, for some $\delta=\delta(\mathbf{x})>0$, that

$$
\beta_{-}(\mathbf{h})-\varepsilon \leq \frac{f(\mathbf{x}+t \mathbf{h})-f(\mathbf{x})}{t} \leq-\beta_{-}(-\mathbf{h})+\varepsilon,
$$

whenever $0<|t|<\delta(\mathbf{x})$. That is,

$$
|[f(\mathbf{x}+t \mathbf{h})-f(\mathbf{x})] / t| \leq \max \left\{\left|\beta_{-}(\mathbf{h})\right|,\left|\beta_{-}(-\mathbf{h})\right|\right\}+\varepsilon \leq L .
$$

Let $\mathbf{y} \in V$. By compactness of the line segment from $\mathbf{x}$ to $\mathbf{y}$, there is a finite sequence $\mathbf{x}_{i}, \mathbf{y}_{i}$ such that

$$
\mathbf{x}=\mathbf{x}_{0}<\mathbf{y}_{0}<\mathbf{x}_{1}<\ldots<\mathbf{y}_{n-1}<\mathbf{x}_{n}=\mathbf{y}
$$


with $\mathbf{y}_{i} \in\left(\mathbf{x}_{i}-\delta\left(\mathbf{x}_{i}\right), \mathbf{x}_{i}+\delta\left(\mathbf{x}_{i}\right)\right)$ and the segments $\left(\mathbf{x}_{i}-\delta\left(\mathbf{x}_{i}\right), \mathbf{x}_{i}+\delta\left(\mathbf{x}_{i}\right)\right)$ covering the line segment from $\mathbf{x}$ to $\mathbf{y}$. Hence

$$
\begin{aligned}
|f(\mathbf{y})-f(\mathbf{x})| & \leq \sum_{i=0}^{n-1}\left|f\left(\mathbf{x}_{i+1}\right)-f\left(\mathbf{y}_{i}\right)\right|+\sum_{i=0}^{n-1}\left|f\left(\mathbf{x}_{i+1}\right)-f\left(\mathbf{y}_{i}\right)\right| \\
& \leq L \sum_{i=0}^{n-1}\left|\mathbf{x}_{i+1}-\mathbf{y}_{i}\right|+L \sum_{i=0}^{n-1}\left|\mathbf{x}_{i+1}-\mathbf{y}_{i}\right| \leq L|\mathbf{y}-\mathbf{x}| .
\end{aligned}
$$

Thus $f$ has Lipschitz constant $L$ on any line segment from $\mathbf{x}$ to $\mathbf{y}$ lying in $V$. This establishes the theorem, since $\mathbf{h}$ was arbitrary.

Corollary 4. Let $f: \mathbb{R}^{N} \rightarrow \mathbb{R}$ in $\boldsymbol{W N T}$ be subadditive. If $\beta_{-}(\mathbf{x})$ is finite for all $\mathbf{x}$, then $f$ is absolutely continuous.

Our next result is inspired by the fundamental result of regular variation, the uniform convergence theorem ([BGT, Section 1.2] and BOst1]). As there, the two basic cases are for $f$ measurable and Baire. It seems that the result is new, even for these cases.

Theorem 9. Let $f: \mathbb{R}^{N} \rightarrow \mathbb{R}$ in $\boldsymbol{W N T}$ be subadditive. If $\beta_{-}(\mathbf{x})$ is finite for all $\mathbf{x}$, then the convergence

$$
F(\mathbf{x}):=\lim _{s \rightarrow+\infty} \frac{f(s \mathbf{x})}{s}, \text { for } \mathbf{x} \in \mathbb{R}^{N}
$$

is uniform on compacts.

Proof. Recall that, for all $t>0$, we have $F(\mathbf{x}) \leq f(t \mathbf{x}) / t$, and, according to the First Limit Theorem, for each $\varepsilon>0$, there is $s=s(\mathbf{x})$ such that, for $s>s(\mathbf{x})$,

$$
F(\mathbf{x}) \leq f(s \mathbf{x}) / s \leq F(\mathbf{x})+\varepsilon
$$

Suppose the theorem is false. Then, for some $\varepsilon>0$, there are $s_{n}>n$ and $\mathbf{x}_{n}$, with $\left\|\mathbf{x}_{n}\right\|=1$, such that $f\left(s_{n} \mathbf{x}_{n}\right) / s_{n}>F\left(\mathbf{x}_{n}\right)+3 \varepsilon$. Since $\left\|\mathbf{x}_{n}\right\|=1$, we may assume that $\mathbf{x}_{n} \rightarrow \mathbf{x}_{0}$. By continuity of $F$ at $\mathbf{x}_{0}$, we can restrict $n$ to be so large that $F\left(\mathbf{x}_{n}\right)>F\left(\mathbf{x}_{0}\right)-\varepsilon$. We then have

$$
f\left(s_{n} \mathbf{x}_{n}\right) / s_{n}>F\left(\mathbf{x}_{0}\right)+2 \varepsilon
$$

According to the limit theorem, we may choose $t_{0}$ so that, for $t>t_{0}$,

$$
F\left(\mathbf{x}_{0}\right) \leq f\left(t \mathbf{x}_{0}\right) / t \leq F\left(\mathbf{x}_{0}\right)+\varepsilon .
$$

By the last theorem, for any fixed $L$ satisfying (4.2), we may by the Lipschitz condition choose $N$ so large that, for $n>N$,

$$
\left|\frac{f\left(s_{n} \mathbf{x}_{n}\right)-f\left(s_{n} \mathbf{x}_{0}\right)}{s_{n}}\right| \leq L\left|\mathbf{x}_{n}-\mathbf{x}_{0}\right| \leq \varepsilon
$$

So for all large enough $n$, we obtain a contradiction to (4.3), namely

$$
\frac{f\left(s_{n} \mathbf{x}_{n}\right)}{s_{n}} \leq \frac{f\left(s_{n} \mathbf{x}_{0}\right)}{s_{n}}+\varepsilon \leq F\left(\mathbf{x}_{0}\right)+2 \varepsilon
$$

Example 2. For $f$ subadditive and also sublinear, Berz's Theorem yields

$$
F(\mathbf{x})=f(t \mathbf{x}) / t, \text { for } t>0,
$$

so here convergence to the limit $F$ is trivially uniform on compact sets. 


\section{REFERENCES}

[BD] F. Bernstein and G. Doetsch, Zur Theorie der konvexen Funktionen, Math. Ann. 76 (1915), 514-526. MR1511840

[Berz] E. Berz, Sublinear functions on $\mathbb{R}$, Aeq. Math. 12 (1975), 200-206. MR.0387862(52:8700)

[BG1] N. H. Bingham and C. M. Goldie, Extensions of regular variation, I: Uniformity and quantifiers, Proc. London Math. Soc., (3) 44 (1982), 473-496. MR656246 (83m:26004a)

[BGT] N. H. Bingham, C. M. Goldie, and J. L. Teugels, Regular variation, Encycl. Math. Appl. 27, Cambridge University Press, Cambridge, 1987. MR898871 (88i:26004)

[BOst1] N. H. Bingham and A. J. Ostaszewski, Foundations of Regular Variation, CDAM Research Report Series, LSE-CDAM-2006-22.

[BOst3] N. H. Bingham and A. J. Ostaszewski, Beyond the Theorems of Steinhaus and Ostrowski: Combinatorial Versions, LSE-CDAM Report, LSE-CDAM-2007-15.

[BOst4] N. H. Bingham and A. J. Ostaszewski, Beyond Lebesgue and Baire: Generic Regular Variation, LSE-CDAM Report, LSE-CDAM-2007-16.

[BOst11] N. H. Bingham and A. J. Ostaszewski, Duality and the Kestelman-Borwein-Ditor Theorem, LSE-CDAM Report, LSE-CDAM-2007-29.

[CsEr] I. Csiszár and P. Erdös, On the function $g(t)=\lim \sup _{x \rightarrow \infty}(f(x+t)-f(x))$, Magyar Tud. Akad. Kut. Int. Közl. A 9 (1964), 603-606.

[Dal] D. J. Daley, Upper bounds for the renewal function via Fourier methods, Annals of Probability 6 (1987), 876-884. MR0494547 (58:13388)

[Fell] W. Feller, An introduction to probability theory and its applications, Volume II, 2nd ed., Wiley, 1971. MR0270403(42:5292)

[HLP] G. H. Hardy, J. E. Littlewood, and G. Pólya, Inequalities. Reprint of the 1952 edition. Cambridge Mathematical Library. Cambridge University Press, Cambridge, 1988. MR.944909 (89d:26016)

[HP] E. Hille and R. S. Phillips, Functional analysis and semi-groups, rev. ed., American Mathematical Society Colloquium Publications, vol. 31. American Mathematical Society, Providence, RI, 1957. MR0089373 (19:664d)

[Kech] A. S. Kechris, Classical descriptive set theory , Graduate Texts in Mathematics 156, Springer, 1995. MR.1321597 (96e:03057)

[King] J. F. C. Kingman, Subadditive processes, É cole d'Été de Probabilités de Saint-Flour, V1975, pp. 167-223. Lecture Notes in Math., Vol. 539, Springer, Berlin, 1976. MR0438477 (55:11388)

[Kucz] M. Kuczma, An introduction to the theory of functional equations and inequalities. Cauchy's functional equation and Jensen's inequality, PWN, Warsaw, 1985. MR788497 (86i:39008)

[Lig] T. M. Liggett, Interacting particle systems, Springer, 1985/2005. MR776231 (86e:60089)

[MatŚw] J. Matkowski and T. Świątkowski, On subadditive functions, Proc. Amer. Math. Soc., 119 (1993), 187-197. MR.1176072 (93k:26002)

[Rock] R. T. Rockafellar, Convex analysis, Princeton University Press, 1970. MR 0274683 $(43: 445)$

[Ros] R. A. Rosenbaum, Sub-additive functions, Duke Math. J. 17 (1950), 227-247. MR0036796(12:164a)

Department of Mathematics, Imperial College London, South Kensington, London SW7 2AZ, UNITED Kingdom

E-mail address: n.bingham@ic.ac.uk

Department of Mathematics, London School of Economics, Houghton Street, LonDON WC2A 2AE, United Kingdom

E-mail address: a.j.ostaszewski@lse.ac.uk 